\title{
A RECIPROCITY THEOREM FOR TENSOR PRODUCTS OF GROUP REPRESENTATIONS
}

\author{
CALVIN C. MOORE ${ }^{1}$ AND JOE REPKA ${ }^{2}$
}

\begin{abstract}
Let $G$ be a type I separable locally compact group. By studying a representation of $G \times G \times G$ we show that a measure class $\lambda$ on $G \times G \times$ $G$ which describes the decompositions of tensor products is invariant under permutations, and that the multiplicity $n\left(\pi_{1}, \pi_{2}, \pi_{3}\right)$ of $\bar{\pi}_{3}$ in $\pi_{1} \otimes \pi_{2}$ is a symmetric function of its variables up to a $\lambda$ null set.
\end{abstract}

1. Introduction. Suppose $K$ is a compact group and $\pi_{1}$ and $\pi_{2}$ are unitary representations of $K$. Then it is well known that the multiplicity of the trivial representation in $\pi_{1} \otimes \pi_{2}$ equals the intertwining number of $\pi_{1}$ and $\bar{\pi}_{2}$ (the bar means contragredient). Now let $\pi_{1}, \pi_{2}, \pi_{3}$ be three irreducible representations of $K$, and consider $\pi_{1} \otimes \pi_{2} \otimes \pi_{3}$. By the above remarks, we see that the multiplicity of the trivial representation in this triple tensor product equals the multiplicity of $\bar{\pi}_{3}$ in $\pi_{1} \otimes \pi_{2}$, or that of $\bar{\pi}_{2}$ in $\pi_{1} \otimes \pi_{3}$, or that of $\bar{\pi}_{1}$ in $\pi_{2} \otimes \pi_{3}$. Thus if we define $n\left(\pi_{1}, \pi_{2}, \pi_{3}\right)$ to be the multiplicity of $\bar{\pi}_{3}$ in $\pi_{1} \otimes \pi_{2}$, the above shows that $n$ is a symmetric function of its three variables. This symmetry is the reciprocity result which we propose to generalize to noncompact groups. The formulation is inspired by Mackey's formulation of the Frobenius reciprocity theorem in [2], and our proof employs the same techniques. In fact, we shall se that what is involved in general is not only a function $n$ on $\hat{G} \times \hat{G} \times \hat{G}$ which will be symmetric in a suitable almost everywhere sense, but also there is a natural measure class on $\hat{G} \times \hat{G} \times \hat{G}$ which we shall prove to be invariant under permutations of the factors. For simplicity we stick to type I groups, as is virtually implied by the last sentence; however, it is evident that our method of proof yields an analogous kind of reciprocity statement quite generally.

If $G$ is the group $\mathrm{SL}_{2}(R)$ where the decompositions of tensor products are known completely, see [3], one may verify the above result directly. However, if $G$ is the group $\mathrm{SL}_{2}(k)$ with $k$ nonarchimedian, the reciprocity theorem which we establish provides new and nontrivial information on the decomposition of tensor products, which will be part of a forthcoming paper by the second-named author. In general the reciprocity statement appears quite useful in that one may know how to decompose some tensor products and

Received by the editors October 28, 1976.

AMS (MOS) subject classifications (1970). Primary 22D10, 22E45, 43A65.

'Supported in part by NSF Gränt MPS 74-19876.

${ }^{2}$ Supported in part by NSF Grant MCS 76-11376. 
not others. In such cases, the known results may be combined with the reciprocity theorem to help decompose other tensor products. Thus this theorem seems to be of some independent interest aside from the specific application noted above.

2. The reciprocity theorem. Let $G$ be a type I separable locally compact group, with unitary dual $\hat{G}$, and let $\mu_{G}$ be Plancherel measure on $G$. For any $\pi_{1}, \pi_{2} \in G$, the tensor product $\pi_{1} \otimes \pi_{2}$ may be decomposed as a direct integral of irreducible representations with multiplicities as

$$
\pi_{1} \otimes \pi_{2}=\int_{G} n\left(\pi_{1}, \pi_{2}, \bar{\pi}_{3}\right) \pi_{3} d \mu_{\pi_{1}, \pi_{2}}\left(\pi_{3}\right)
$$

The measure $\mu_{\pi_{1}, \pi_{2}}$ on $\hat{G}$ is well defined up to equivalence and $n\left(\pi_{1}, \pi_{2}, \cdot\right)$ is defined up to a $\mu_{\pi_{1}, \pi_{2}}$ null set. We let $\bar{\mu}_{\pi_{1}, \pi_{2}}$ be the transform of $\mu_{\pi_{1}, \pi_{2}}$ under $\pi_{3} \rightarrow \bar{\pi}_{3}$, and then we form a measure $\lambda$ on $\hat{G} \times \hat{G} \times \hat{G}$ by integrating the fiber measures $\bar{\mu}_{\pi_{1}, \pi_{2}}$ on $\hat{G}$ over the base measure $\mu_{G} \times \mu_{G}$ on $\hat{G} \times \hat{G}$ (viewed as the first two coordinates). The measure $\lambda$ is, of course, precisely the measure $\alpha$ of Theorem 5.1 of [2] in the case when the larger group is $G \times G$ and its subgroup is $G$, embedded as the diagonal, except that $\alpha$ has been transformed by the map $\pi_{3} \rightarrow \bar{\pi}_{3}$ in the third variable. The multiplicity function $n$ on $\hat{G} \times \hat{G} \times \hat{G}$ which is defined $\lambda$-almost everywhere is as in [2] also. The desired reciprocity statement is the following statement.

THEOREM. Let $G$ be type I; then the class of the measure $\lambda$ on $\hat{G} \times \hat{G} \times \hat{G}$ is invariant under permutations of the three factors and, up to a $\lambda$ null set, the function $n$ is invariant under permutations of the three factors.

Proof. The proof is achieved rather as in [2]. We form a representation $\sigma$ of the type I group $G \times G \times G$ determined canonically by the measure $\lambda$ and multiplicity function $n$. Then we have that

$$
\sigma=\int_{\hat{G} \times \hat{G}} \int_{\hat{G}} n\left(\pi_{1}, \pi_{2}, \pi_{3}\right) \pi_{1} \times \pi_{2} \times \pi_{3} d \bar{\mu}_{\pi_{1}, \pi_{2}}\left(\pi_{3}\right) d \mu_{G}\left(\pi_{1}\right) d \mu_{G}\left(\pi_{2}\right)
$$

where $\pi_{1} \times \pi_{2} \times \pi_{3}$ refers to the outer tensor product. The desired results will follow by uniqueness of direct integral decompositions if we can show that the class of the representation $\sigma$ above is invariant under the outer automorphisms of $G \times G \times G$ induced by permutations of the three factors.

Now let $\tau$ be the two-sided regular representation of $G \times G$ on $L^{2}(G \times G) ; \tau$ is a representation of $(G \times G) \times(G \times G)$ and we let $\sigma^{\prime}$ be its restriction to $G \times G \times G$ viewed as the subgroup $\{(s, t, r, r)\}$. It is known that $\tau$ has a direct integral decomposition

$$
\tau=\int_{\hat{G} \times \hat{G}}\left(\pi_{1} \times \pi_{2}\right) \times\left(\bar{\pi}_{1} \times \bar{\pi}_{2}\right) d \mu_{G}\left(\pi_{1}\right) d \mu_{G}\left(\pi_{2}\right),
$$

where we identify $(G \times G)^{\wedge}$ with $\hat{G} \times \hat{G}$ and Plancherel measure with the product of Plancherel measures. It follows that $\sigma^{\prime}$ has the form 


$$
\sigma^{\prime}=\int_{\hat{G} \times \hat{G}}\left(\pi_{1} \times \pi_{2}\right) \times\left(\int n\left(\bar{\pi}_{1}, \bar{\pi}_{2}, \bar{\pi}_{3}\right) \pi_{3} d \mu_{\bar{\pi}_{1}, \bar{\pi}_{2}}\left(\pi_{3}\right)\right) d \mu_{G}\left(\pi_{1}\right) d \mu_{G}\left(\pi_{2}\right),
$$

and a simple transformation of variables shows that $\sigma^{\prime}$ is equivalent to $\sigma$. Our result now follows from the following observation.

Proposition. The class of the representation $\sigma^{\prime}$ is invariant under permutations of the three factors.

Proof. The representation $\sigma^{\prime}$ is realized on $L^{2}(G \times G)$ in the form

$$
\left(\sigma^{\prime}\left(g_{1}, g_{2}, g_{3}\right) f\right)\left(t_{1}, t_{2}\right)=\Delta\left(g_{3}\right) f\left(g_{1}^{-1} t_{1} g_{3}, g_{2}^{-1} t_{2} g_{3}\right)
$$

where $\Delta$ is the modular function of $G$. The class of $\sigma^{\prime}$ is visibly invariant under transposition of the first two factors. Then we note that the unitary operator $(U f)(s, t)=\Delta\left(s^{-1}\right) f\left(s^{-1}, t s^{-1}\right)$ implements a unitary equivalence of $\sigma^{\prime}$ and $\sigma^{\prime}$ transformed by transposition of the first and third variables. Since these two transpositions generate all permutations, we are done.

3. Examples and remarks. As noted in the introduction, one knows the decomposition of tensor products of representations of the group $\mathrm{SL}_{2}(R)$. The invariance of $\lambda$ may be verified and the invariance of $n$ as asserted in the theorem holds for all $\left(\pi_{1}, \pi_{2}, \pi_{3}\right)$ except possibly when one or more of the $\pi_{i}$ 's is in the complementary series or is one of the irreducible components of the reducible principal series representation. It is interesting that the invariance of $n$ fails to hold everywhere. Another example of interest is that of a simply connected nilpotent Lie group $N$. Here one knows $\hat{N}$ and how tensor products decompose [1]. We shall describe the measure $\lambda$ on $\hat{N} \times \hat{N} \times \hat{N}$ very concretely. Indeed, by [1], $\hat{N} \times \hat{N} \times \hat{N}$ is a quotient space of $V=\mathfrak{n}^{*} \oplus$ $\mathrm{n}^{*} \oplus \mathrm{n}^{*}$ where $\mathrm{n}^{*}$ is the linear dual of the Lie algebra $\mathrm{n}$ of $N$, and we describe $\lambda$ by describing a measure $\lambda_{0}$ on $V$ whose class projects onto $\lambda$. Indeed, $\lambda_{0}$ is concentrated on the subspace $V_{0}$ of $V$ consisting of triples $\left(f_{1}, f_{2}, f_{3}\right)$ with $f_{1}+f_{2}+f_{3}=0$, and $\lambda_{0}$ is just Lebesgue measure on two subspaces. The invariance of the class of $\lambda$ under permutations of the three factors is now quite evident. The function $n$ can be described by describing it on $V_{0}$ instead and, indeed, $n\left(f_{1}, f_{2}, f_{3}\right)$ is a constant almost everywhere on $V_{0}$, and the constant is either $\infty$ or 1 .

If $G$ fails to be type I, then, of course, the Proposition above is still valid so one expects some sort of reciprocity theorem. The ingredient in the formulation would be a standard measure class on the quasi-dual (quasiequivalence classes of primary representations) of $G \times G \times G$ which would be invariant under permutations. It would, however, no longer make sense to talk about multiplicities. We omit the details.

\section{REFERENCES}

1. A. A. Kirillov, Unitary representations of nilpotent Lie groups, Uspehi Mat. Nauk 17 (1962), no. 4 (106), 57-110 = Russian Math. Surveys 17 (1962), no. 4, 53-104. MR 25 \# 5396. 
2. G. W. Mackey, Induced representations of locally compact groups, II. The Frobenius reciprocity theorem, Ann. of Math. (2) 58 (1953), 193-221. MR 15, 101.

3. J. Repka, Tensor products of unitary representations of $\mathrm{SL}_{2}(R)$, Bull. Amer. Math. Soc. 82 (1976), 930-932.

Departmignt of Mathematics, University of Calmodnia, Berkeley, Calupornia 94720

Departugant of Mathimatics, Princeton Universtty, Prnnceton, New Jersey 08540 